10-1999

\title{
Reactive Infiltration of Silicon Melt Through Microporous Amorphous Carbon Preforms
}

P. Sangsuwan

BAMJWathmondrddraideditional works at: https://engagedscholarship.csuohio.edu/encbe_facpub

Surehitrafitherelatorials Science and Engineering Commons

Aleweldodstatedingiveritijs work benefit you? Let us know!

Publisher's Statement

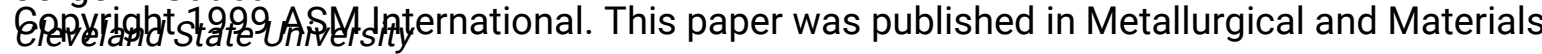

Transactions B: Process Metallurgy and Materials Processing Science, Vol. 30, Issue 5, pp.

33 Singh and is made available as an electronic reprint with the permission of ASM International. NYMA NASA Lewis Group, Inc.,

One print or electronic copy may be made for personal use only. Systematic or multiple

Peppockesesiom, distribution to multiple locations via electronic or other means, duplications of any

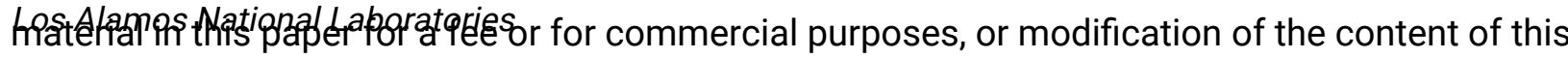
paper are prohibited.

Available on publisher's site at: http://www.springerlink.com/content/q08846334u232027/.

\section{Original Citation}

Sangsuwan, P; Tewari, SN; Gatica, JE; Singh, M; Dickerson, R. (1999). Reactive Infiltration of Silicon Melt Through Microporous Amorphous Carbon Preforms. Metallurgical and Materials Transactions B: Process Metallurgy and Materials Processing Science 30, 933-944.

\section{Repository Citation}

Sangsuwan, P.; Tewari, Surendra N.; Gatica, Jorge E.; Singh, M.; and Dickerson, R., "Reactive Infiltration of Silicon Melt Through Microporous Amorphous Carbon Preforms" (1999). Chemical \& Biomedical Engineering Faculty Publications. 16.

https://engagedscholarship.csuohio.edu/encbe_facpub/16

This Article is brought to you for free and open access by the Chemical \& Biomedical Engineering Department at EngagedScholarship@CSU. It has been accepted for inclusion in Chemical \& Biomedical Engineering Faculty Publications by an authorized administrator of EngagedScholarship@CSU. For more information, please contact library.es@csuohio.edu. 


\title{
Reactive Infiltration of Silicon Melt through Microporous Amorphous Carbon Preforms
}

\author{
P. SANGSUWAN, S.N. TEWARI, J.E. GATICA, M. SINGH, and R. DICKERSON
}

\begin{abstract}
The kinetics of unidirectional capillary infiltration of silicon melt into microporous carbon preforms has been investigated as a function of the pore morphology and melt temperature. The infiltrated specimens showed alternating bands of dark and bright regions, which corresponded to the unreacted free carbon and free silicon regions, respectively. The decrease in the infiltration front velocity for increasing infiltration distances is in qualitative agreement with the closed-form solution of capillaritydriven fluid flow through constant-cross-section cylindrical pores. However, drastic changes in the thermal response and infiltration front morphologies were observed for minute differences in the preform's microstructure. This suggests the need for a dynamic percolation model that would account for the exothermic nature of the silicon-carbon chemical reaction and the associated pore-closing phenomenon.
\end{abstract}

\section{INTRODUCTION}

EXOTHERMIC reactions between a porous matrix and an infiltrating melt provide a more economic alternative for synthesizing many ceramics, intermetallics, and composites. The manufacture of reaction-bonded silicon carbide can be attained by capillary infiltration of molten silicon ${ }^{[1]}$ through a carbon-containing body. These carbon preforms typically contain a large amount of $\mathrm{SiC}$ grains as inert fillers. The exothermic reaction between silicon and carbon results in the formation of silicon-carbide particles that bond the previously existing $\mathrm{SiC}$ grains. In another approach, carbonaceous materials, such as carbon fiber tow, carbon fiber cloth, or felt, are infiltrated by molten silicon in vacuum to form $\mathrm{Si} / \mathrm{SiC}$ (silicon-carbide reinforced silicon composites). ${ }^{[1]}$ It has recently been demonstrated that infiltration of cast microporous carbon preforms by silicon melt can be used to fabricate high-density near net-shaped silicon-carbide components at significantly reduced cost. ${ }^{[2,3]}$ Components fabricated by this technique are expected to find applications as gas turbine engine components and commercial combustion nozzles, where their refractoriness (high-temperature strength), good oxidation resistance, high thermal conductivity, low density, and adequate toughness can be fully exploited. However, successful commercial exploitation of this technique requires a quantitative understanding of the various steps involved in the process.

The siliconization mechanism has been extensively studied via experiments on isolated carbon fibers or plates. ${ }^{[4-9]}$ Since molten silicon wets carbon, it wicks up into the microporous carbon preform when it is brought in contact with the preform, thus converting carbon into silicon carbide. It

P. SANGSUWAN, formerly Graduate Student, Department of Chemical Engineering, Cleveland State University, is a Process Engineer, with $3 \mathrm{M}$ Thailand Limited, Bangkok, Thailand 10520. S.N. TEWARI, Professor, and J.E. GATICA, Associate Professor, are with the Department of Chemical Engineering, Cleveland State University, Cleveland, OH 44115-2426. M. SINGH, Senior Research Engineer, is with NYMA, Inc., Lewis Research Center Group, Cleveland, OH 44135. R. DICKERSON, formerly Senior Research Engineer, NYMA Inc., is Senior Scientist, Los Alamos National Laboratories, Los Alamos, NM 87545.

Manuscript submitted April 9, 1998. was initially believed that after a thin film of silicon carbide formed on the carbon surface, diffusion of silicon or carbon through the solid was responsible for further conversion. ${ }^{[4,5]}$ Grain-boundary diffusion was initially invoked to explain the fast conversion kinetics that were generally observed. It was later proposed that the initial silicon-carbide layer spalls off because of the volume increase due to the reaction, thereby exposing a fresh carbon surface to the liquid silicon. Further conversion occurred by dissolution of carbon into the molten silicon at higher temperatures. Silicon carbide then precipitated in regions of lower temperature. ${ }^{[6,7,8]}$

However, there has been very little effort to study the most important step in the conversion process: the process of silicon infiltration through the porous carbon preforms. Unlike infiltration processes where there is no reaction between the porous preforms and the infiltrating liquid, the reaction between the infiltrating silicon melt and carbon results in a reduced pore size. There is an approximate 58 pct volume increase for each mole of silicon carbide formed. This reduces the permeability of the preform and decreases the infiltration velocity. Therefore, if the initial pore size of the preform is small, the silicon infiltration can prematurely stop, resulting in an incomplete infiltration. This phenomenon is frequently referred to as "choking." Alternatively, if channel sizes are too large, the preform would be fully infiltrated, but unreacted silicon may remain in the component.

Messner and Chiang ${ }^{[9]}$ formulated an analytical model based on Darcy's law. ${ }^{[10]}$ This model incorporated a varying permeability. The model was used to predict the infiltration behavior of silicon through graphite preforms. However, no measurements of the infiltration kinetics were carried out. There is only one study reported in the literature ${ }^{[11]}$ where an attempt has been made to measure the infiltration front velocity as a function of temperature. Einse ${ }^{[11]}$ exposed thin carbon tapes, fabricated by two different techniques, to a silicon melt to determine the infiltration kinetics. Thermocouples were inserted at different lengths along the tapes to monitor the propagation of the infiltrating reaction front. These experiments, however, do not accurately represent infiltration through the porous preform because silicon melt would preferentially wet and traverse along the outer surface 

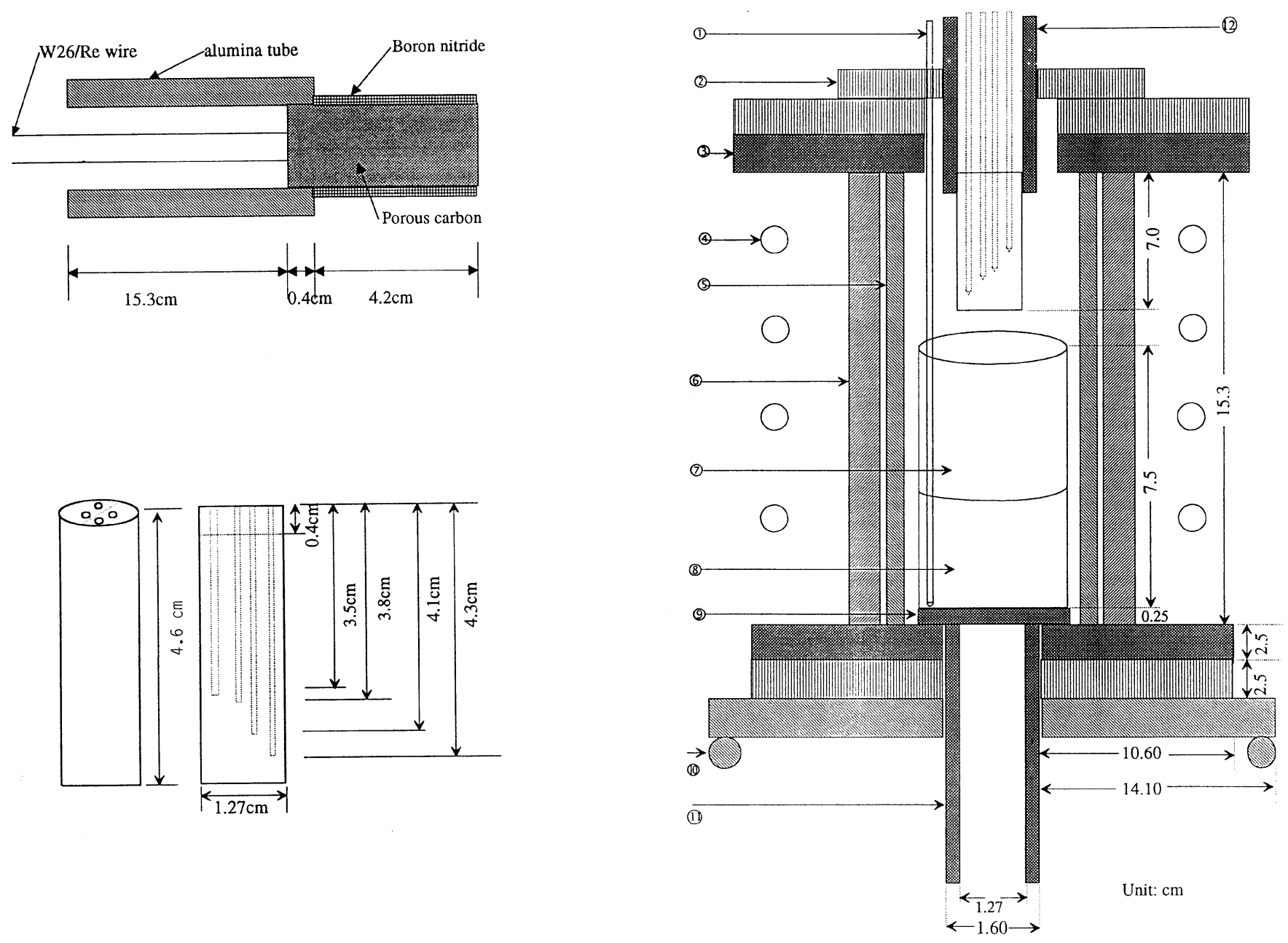

Fig. 1-Schematic view of the experimental apparatus for measuring silicon infiltration kinetics.

of the tapes where there is no resistance to the melt flow, rather than through the interior torturous channels.

The purpose of this research was to examine the interplay between chemical kinetics and capillary flow for silicon infiltration into microporous carbon preforms. The investigation focused on the effect of pore structure and processing conditions on the infiltration process. Two types of carbon preforms were infiltrated at four different melt temperatures $(1703,1743,1788$, and $1853 \mathrm{~K})$. The infiltration dynamics were measured using thermocouples embedded in the preforms.

\section{EXPERIMENTAL PROCEDURE}

\section{A. Microporous Carbon Preforms}

The microporous amorphous carbon preforms used in this study were made from a mixture of furfuryl alcohol resin, di-ethylene and tri-ethylene glycols, and $p$-toluene sulfonic acid. This mixture was polymerized to form a porous solid polymer. The solid polymer was then heated slowly up to $970 \mathrm{~K}$ in a flowing argon atmosphere, which resulted in the production of microporous carbon preforms. Preforms with a range of pore sizes were obtained by varying the composition of the organic mixture. Further details of the preform fabrication can be found elsewhere. ${ }^{[3]}$ The microstructure of the preforms was examined by scanning electron microscopy (SEM) and characterized by mercury porosimetry and permeability determinations.

During mercury porosimetry, the porous preform is immersed in a mercury bath, and the intruded volume of mercury is measured as a function of the applied hydrostatic pressure. This information is then used to obtain the incremental intrusion volume (per unit weight) of the porous specimen as a function of the equivalent diameter of cylindrical pores. The incremental intrusion area (per unit weight) as a function of the pore diameter is also obtained. These data are then used to obtain the pore size distributions, based either on the pore volumes or the pore areas. The skeletal density is determined from the bulk density and total intruded volume data assuming that the pores are continuous; i.e., no isolated pores exist in the specimen.

Permeability determinations consisted of measuring water-flow rates through the preforms as a function of the applied pressure gradient. Darcy's law ${ }^{[10]}$ was then used to correlate the measurements and obtain the preform permeability.

\section{B. Analysis of Silicon Melt Infiltration}

Figure 1 is a schematic view of the experimental setup used to analyze the process of silicon melt infiltration 
through the porous preforms, as a function of the melt temperature. Four longitudinal closed-end holes were drilled on one end of the cylindrical $1.27-\mathrm{cm}$ diameter by $4.6-\mathrm{cm}$ long carbon preforms. Tungsten-rhenium thermocouples, kept inside twin-bored alumina sheaths, were inserted into these holes, such that their tips would be located at $0.3,0.5,0.8$, and $1.1 \mathrm{~cm}$ from the closed end of the preform. The side surface of the preform was coated with a refractory paste to prevent side infiltration of silicon. The preform was then inserted in an alumina tube (12) which was rigidly fixed to the top insulator (2). An induction power supply (4) was used to heat the graphite susceptor (5), which heated the preform and the silicon melt contained in a quartz crucible (8). A thermocouple (1) recorded the temperature of the silicon melt. The whole assembly was kept under a vacuumcontrolled atmosphere inside a transparent chamber.

Silicon (99.9995 pct purity) was first melted in a flowing argon atmosphere, and then brought to the desired temperature. The silicon-melt-containing crucible was then raised, bringing the melt level to about $0.5-\mathrm{cm}$ away from the bottom surface of the carbon preform. Once the preform and the melt had reached thermal equilibrium, the crucible was raised until approximately $1.5 \mathrm{~cm}$ of the preform was immersed in the silicon melt to initiate the infiltration process. After approximately 2 minutes, the crucible was lowered, and the induction power was discontinued. The thermal responses of the thermocouples as a function of time, kept in the preform and in the silicon melt, were continuously recorded with the help of a Hewlett Packard data logger. After the infiltrated sample was cooled to room temperature, it was taken out, sectioned, and examined by optical and scanning electron metallography and electron microprobe (EMP) analysis.

\section{RESULTS}

\section{A. Microstructural Characterization of the Preforms}

Figure 2 shows typical microstructures of the two types of microporous carbon preforms used in this study. The carbon struts are bridged with each other, and the inter-strut regions provide an interconnected continuous porosity. The type B preform appears to be more open when compared with type A. The three-dimensional (3-D) network of interconnected pores does not appear to have any anisotropy.

Figure 3 presents the results of porosimetry determinations carried out on two different preforms. The percent intrusion volume (Figure 3(a)), the percent intrusion area (Figure 3(b)) vs the pore diameter, and the corresponding pore size distributions (Figure 3(c)) are shown. Both preforms show a narrow pore size distribution. Their median pore diameters, based either on the pore volume or on the pore surface area, are nearly identical (Table I). A comparison between their skeletal densities, 1.48 and $1.51 \mathrm{~g} \mathrm{~cm}^{-3}$, and the literature-reported density of glassy carbon, $1.5 \mathrm{~g}$ $\mathrm{cm}^{-3}$, suggests that the pore networks in these preforms are interconnected. In the presence of isolated pores, the skeletal density calculated from mercury porosimetry would be smaller than that corresponding to glassy carbon. However, the type $\mathrm{B}$ preform is more porous when compared with the type A, as shown by their fraction porosity, 0.53 vs 0.48 (Table I). The pore surface area per unit pore volume corresponding to the type A preform is approximately 24 pct larger

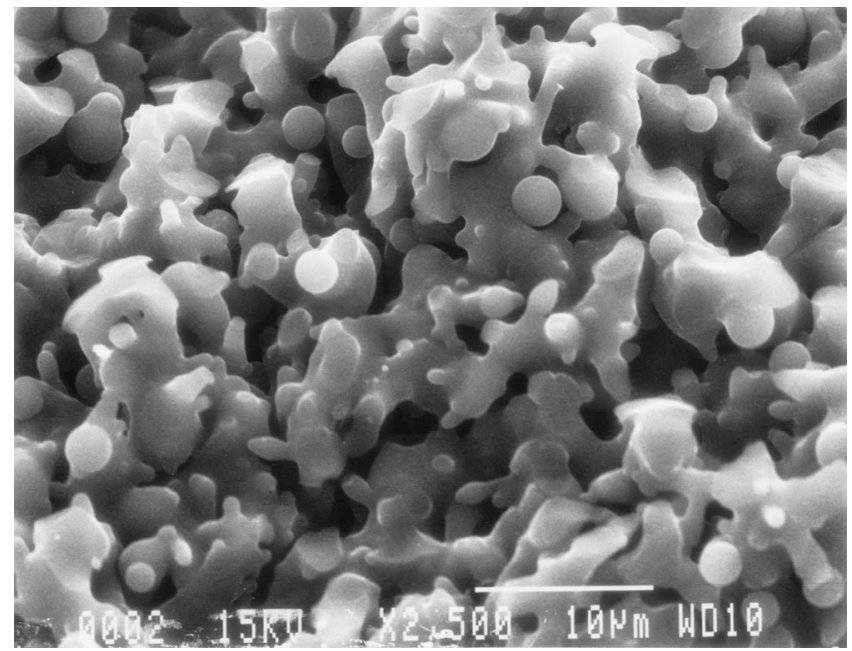

(a)

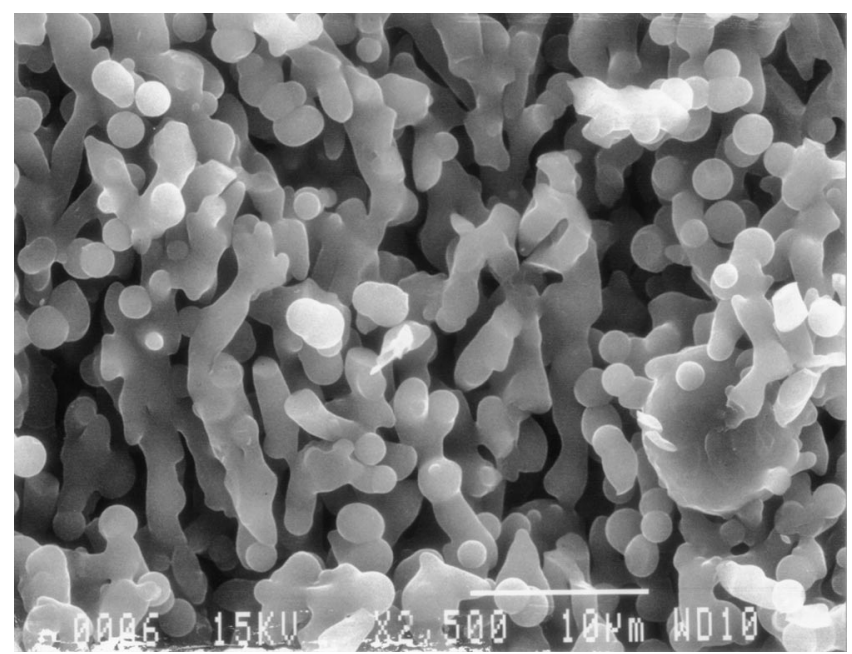

(b)

Fig. 2-Typical microstructure of porous amorphous carbon preform: (a) type A and (b) type B.

than that of preform of type B. The intrusion measurements can also be used to corroborate this characteristic. Indeed, if the ratio between the intrusion volume and the total intrusion area could be used as an estimation of an "effective" pore diameter, the preform A would have a smaller effective pore diameter than the preform B; i.e., 0.11 vs $0.14 \mu \mathrm{m}$.

\section{B. Permeability Analysis of Uninfiltrated Specimens}

The porous medium permeability, $\kappa$, is an overall macroscopic parameter that is widely used to describe fluid flow through porous structures in many engineering applications. For a Boussinesq's fluid, Darcy's law ${ }^{[10]}$ is as follows:

$$
\begin{aligned}
\rho_{f}^{0} \frac{\partial \vec{u}}{\partial t}= & -\nabla p-\frac{\mu_{f}}{\kappa} \vec{u}-\rho_{f}^{0}\left[\beta_{T}\left(T-T^{0}\right)\right. \\
& \left.+\beta_{C}\left(C-C^{0}\right)\right] \vec{g}
\end{aligned}
$$

where $u$ is the fluid velocity, $p$ the pressure, $\rho$ indicates the fluid density, $\mu$ the dynamic viscosity, $\kappa$ represents the permeability of the porous medium, $g$ is the gravitational 
POROSIMETRY ANALYSIS OF MICROPOROUS

CARBON PREFORMS

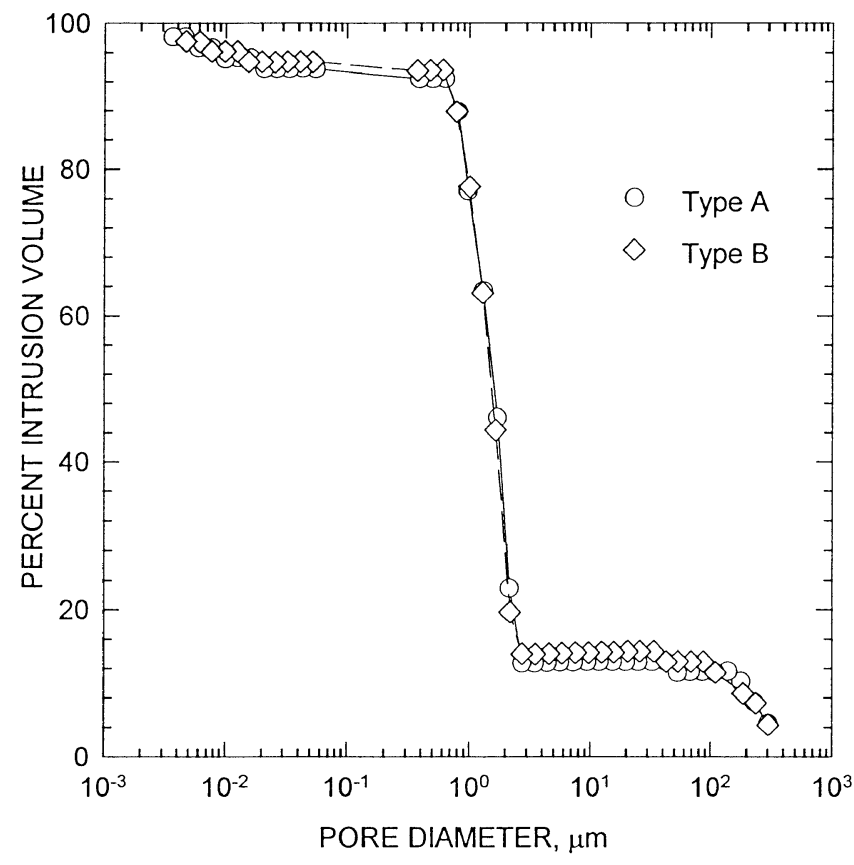

(a)

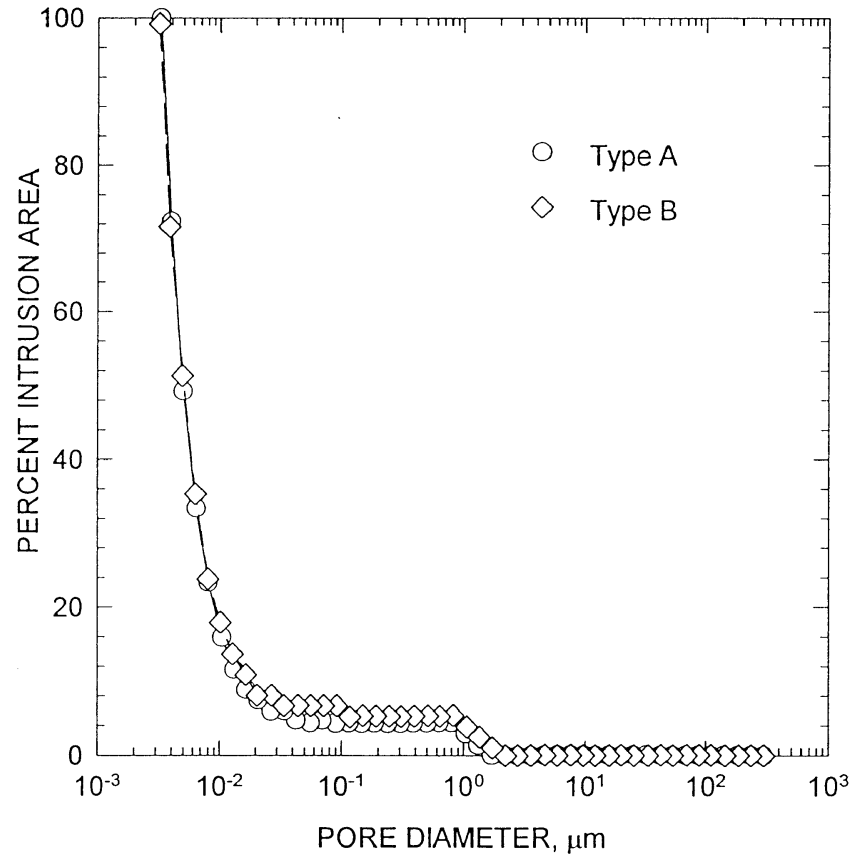

(b)

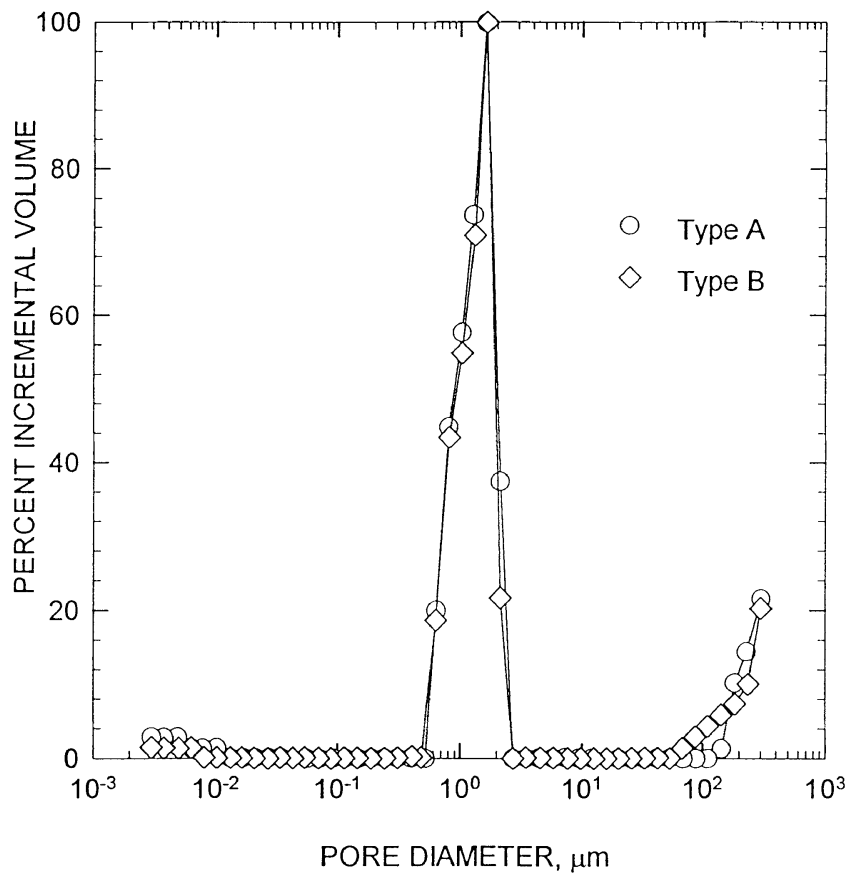

(c)

Fig. 3-Typical pore characteristics of carbon preforms obtained by mercury porosimetry: $(a)$ percent intrusion volume $v s$ pore diameter, $(b)$ percent intrusion area $v s$ pore diameter, and $(c)$ percent incremental volume $v s$ pore diameter.

acceleration, $T$ is the temperature, $C$ indicates concentration, and $\beta$ represents the thermal-expansion coefficient.

Assuming steady-state conditions and neglecting buoyancy effects, for unidirectional flow, Darcy's relationship reduces to the following:

$$
v=-\frac{\kappa \partial p}{\mu_{f} \partial z}
$$

which can be readily solved assuming a linear dependence for the pressure. This relationship can be used to extract the 
Table I. Mercury Porosimetry and Permeability Results for Microporous Carbon Specimens

\begin{tabular}{lcc}
\hline \multicolumn{1}{c}{ Characteristics } & Type A & Type B \\
\hline Median pore diameter, $\mu \mathrm{m}$ & 1.42 & 1.25 \\
Average pore diameter, $\mu \mathrm{m}$ & 1.56 & 1.49 \\
Fraction porosity & 0.48 & 0.53 \\
Bulk density, $\mathrm{g} \mathrm{cm}^{-3}$ & 0.77 & 0.70 \\
Skeletal density, $\mathrm{g} \mathrm{cm}^{-3}$ & 1.48 & 1.51 \\
Pore area per unit pore volume $\times$ & 3.64 & 2.93 \\
$\quad 10^{-7}, \mathrm{~m}^{-1}$ & & \\
"Effective" pore diameter, 4V/A, & 0.11 & 0.14 \\
$\quad \mu \mathrm{m}$ & & \\
Permeability $\times 10^{14}, \mathrm{~m}^{2}$ & $2.29 \pm 0.03$ & $2.70 \pm 0.01$ \\
\hline
\end{tabular}

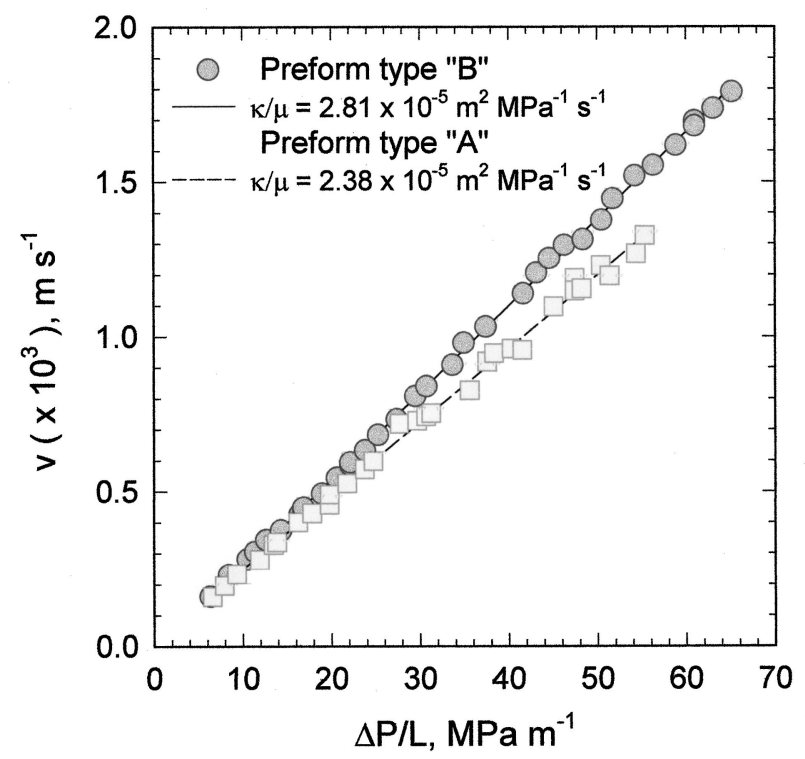

Fig. 4-Permeability determinations for type A and type B microporous carbon preforms.

permeability of the medium from a linear correlation between fluid flow velocity $(v)$ and the applied pressure gradient. Figure 4 shows the permeability measurements corresponding to preforms of type A and B. The goodness of fit of these determinations supports the applicability of the Darcy's law for the preforms used in this study. For a room-temperature viscosity of water of $9.6 \times 10^{-10} \mathrm{MPa} \mathrm{s}$, the permeabilities of the two preforms, $\mathrm{A}$ and $\mathrm{B}$, can be estimated as $2.29 \times 10^{-14}$ and $2.70 \times 10^{-14} \mathrm{~m}^{2}$, respectively (Table I).

\section{Microstructural Analysis of Infiltrated Specimens}

Figure 5 shows typical microstructures after infiltration. Figure 5(a) shows an overall view of a specimen after infiltration. The bottom $1.7 \mathrm{~cm}$ of this preform was dipped in the silicon melt. The white layer is the boron-nitride coating, while the dark layer is the silicon stuck to the boron-nitride coating. This dark layer and the boron-nitride coating underneath came off easily from the reacted specimen. An arrow indicates some silicon, which remained attached to the bottom surface when the preform was withdrawn from the melt.

Figure 5(b) shows longitudinal (parallel to the silicon infiltration direction, indicated by an arrow on the left side) cross sections for type A specimens that were infiltrated at melt temperatures of 1743,1783 , and $1853 \mathrm{~K}$. The dark regions on the top of the micrograph correspond to the uninfiltrated portions of the preform. The infiltrated portions of the preform appear at the bottom of the micrographs. At $1743 \mathrm{~K}$, the type A preform was infiltrated up to a distance of about $0.28 \mathrm{~cm}$ before choking was observed. Instabilities developing at the front were observed with increasing melt temperature. For example, at a melt temperature of $1783 \mathrm{~K}$, the infiltration front is no longer planar; instead, fingers of infiltrated regions protrude ahead of the overall infiltration front. At the highest melt temperature examined in this study, $1853 \mathrm{~K}$, there is hardly any region that would appear to indicate the presence of a stable planar liquid infiltration front. The microstructure consists of a "swirling" pattern of isolated infiltrated regions.

The infiltrated portion of the specimens usually consists of alternating bands of dark and light contrast regions, marked as D and B in Figure 5(b). Higher magnification views from these two regions are shown in Figure 6. The dark regions (Figure 6(a)) consist of silicon-carbide grains (gray contrast), free silicon (white contrast), and unreacted carbon (black contrast). The large amount of unreacted carbon is responsible for the dark appearance of these regions. The bright regions (Figure 6(b)) contain mostly silicon carbide and free silicon, with very little unreacted carbon present.

Figure 7 shows two types of cracks observed in infiltrated specimens. The thermal stresses, resulting from the exothermic nature of the chemical reaction, can produce cracks in portions of the preform that are yet to be infiltrated as well as in the portions that have already been infiltrated. The bright-looking silicon-filled cracks in Figure 7(a) must have formed before the preform was infiltrated with silicon melt, while the dark-looking cracks in Figure 7(b) were generated after the infiltration and reaction.

Figure 8 shows the longitudinal section corresponding to a porous carbon preform type B infiltrated by a silicon melt at $1743 \mathrm{~K}$. For the same melt temperatures, the infiltration distance is significantly larger for the type B material; 0.92 $\mathrm{cm}$ in Figure 8 vs $0.28 \mathrm{~cm}$ in Figure 5(b). The bright and dark bands observed for the type A preforms, at 1743 and $1783 \mathrm{~K}$ (Figure 5(b)), were also observed for the type B preform. However, the fingerlike instability observed for the type A material was not observed in type B material in the temperature range examined in this study (1703 to $1853 \mathrm{~K}$ ).

\section{Thermal Responses during Infiltration}

Typical temperature-time plots recorded by the thermocouple assembly described previously are shown in Figures 9(a) through 10(c). The thermal responses of the thermocouples located at $0.3,0.5,0.8$, and $1.1 \mathrm{~cm}$ from the (bottom) surface of the preforms exposed to the silicon melt are indicated as TC1, TC2, TC3, and TC4, respectively. The thermal response corresponding to the silicon melt is indicated as $\mathrm{Si}$. 


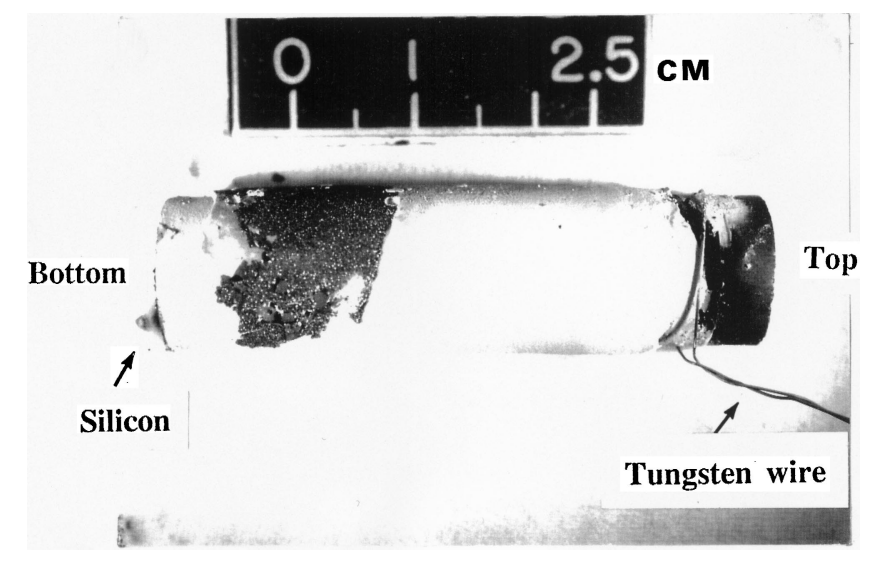

(a)
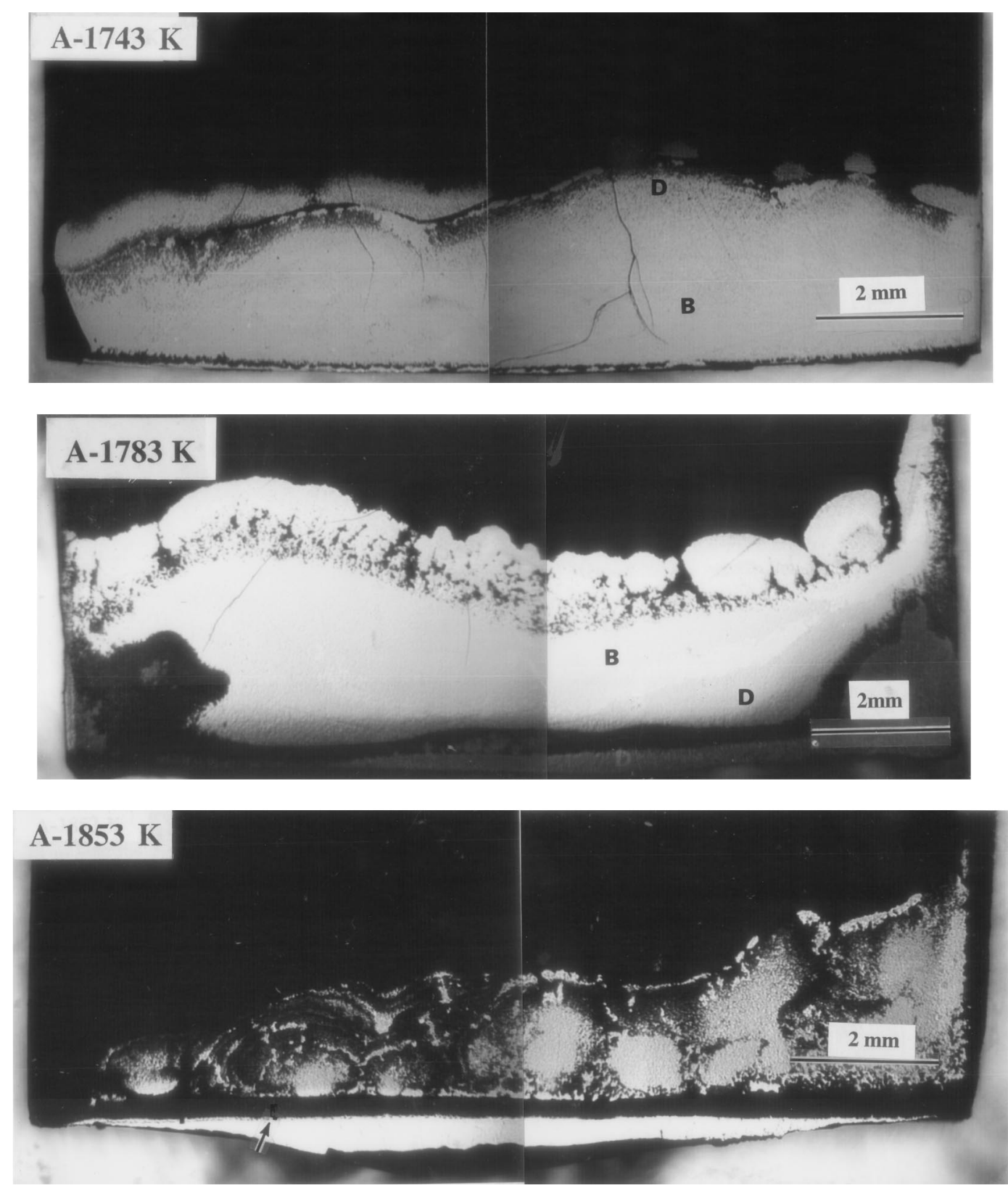

(b)

Fig. 5-Typical microstructure of the silicon-infiltrated specimens. (a) Overall view of an infiltrated specimen. (b) Longitudinal (parallel to the infiltration direction) sections through the infiltrated "A" specimens. The "dark" contrast regions are marked "D," and the "bright" contrast regions are marked 

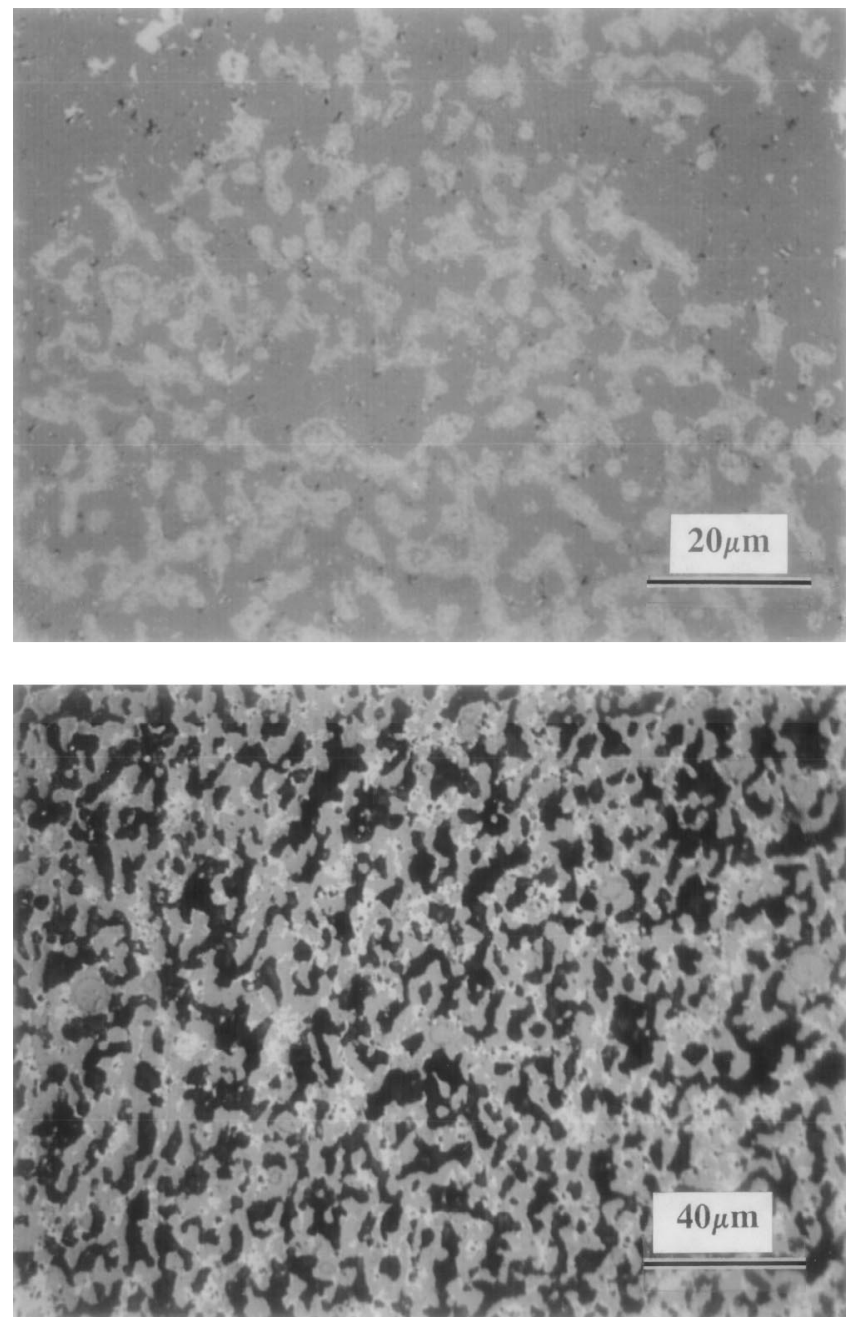

Fig. 6-Higher-magnification views from the dark (D) and bright contrast (B) regions of the infiltrated microstructure shown in Fig. 5(b).

\section{Type A preform}

Figure 9(a) shows the thermal profiles obtained from infiltration experiments carried out with type A preforms. The uncertainty in the temperature values is approximately \pm 6 $\mathrm{K}$. The arrows in the figure (bottom) indicate the different stages of the infiltration experiments. The melt crucible was initially raised at 180 seconds. From 245 to 1655 seconds, the crucible was held stationary for the final adjustment of the power supply to yield the desired melt temperature (1788 $\mathrm{K}$ ), and until thermal equilibrium among the melt, the preform, and the surrounding susceptor is reached. The crucible was then raised at 1835 seconds, with the melt-carbon preform contact occurring at 1853 seconds; further crucible raising resulted in $1.7 \mathrm{~cm}$ of the preform being submerged in the melt. The exothermic reaction between the infiltrating silicon melt and the carbon preform resulted in a steep temperature rise, recorded by all thermocouples. It is worth noticing, however, that despite the highly exothermic reaction, the temperatures recorded by all preform thermocouples are back to the silicon melt temperature within 60 seconds after the initial temperature rise. After about 2 minutes of melt-preform contact, the crucible was pulled down (2015 seconds). The preform was out of the melt at about 2090 seconds, at which time the induction power was switched

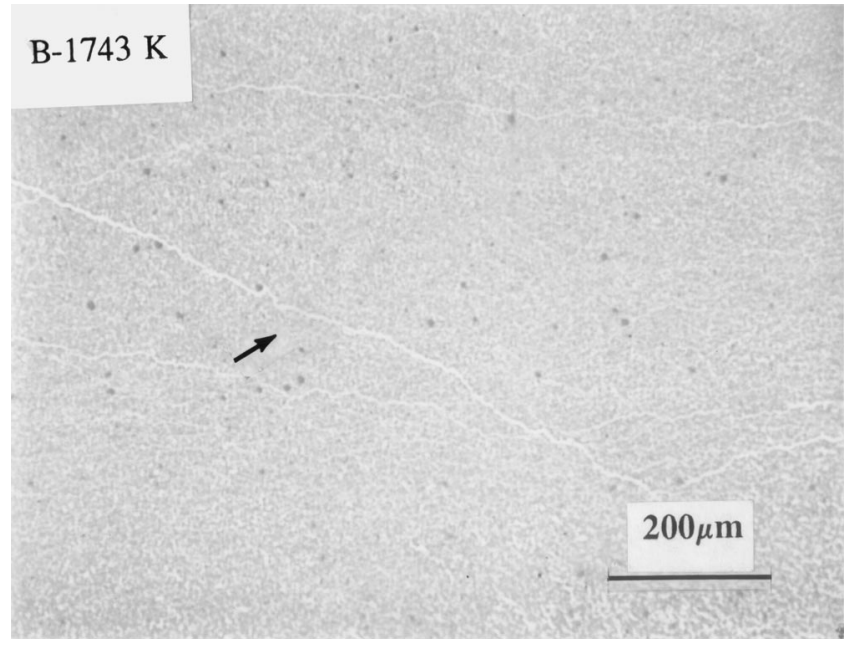

(a)

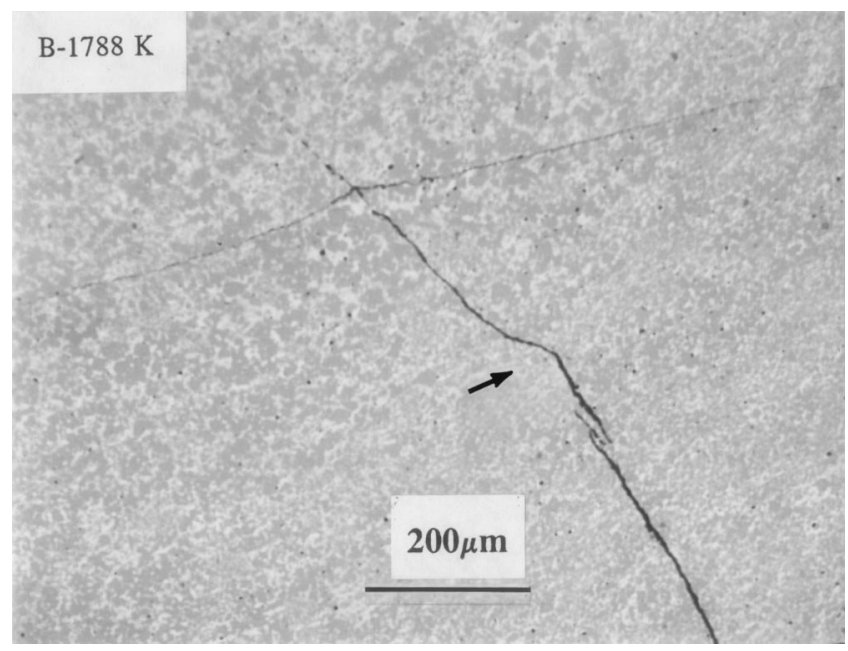

(b)

Fig. 7-Typical cracks observed in the silicon melt-infiltrated specimens: (a) cracks formed prior to infiltration and (b) cracks formed after infiltration and chemical reaction between carbon and silicon.

off. This resulted in a rapid cooling, approximately $50 \mathrm{~K}$ $\mathrm{min}^{-1}$, recorded by all five thermocouples. The isothermal plateau, starting at 2240 seconds, registered by the thermocouple in the silicon melt corresponds to the solidification of silicon $(1687 \mathrm{~K})$. It is interesting to note that the isothermal trend was also indicated by the thermocouples located in the preform, especially TC1, TC2, and to some extent TC3 (Figure 9(c)). This indicates the presence of free silicon in the preform, as it was previously suggested by the microstructural analysis (Figure 6(b)). It also suggests that the silicon infiltration front reached a depth between TC3 and TC4; i.e., an infiltration distance between 0.5 and $0.8 \mathrm{~cm}$. This is in agreement with the metallographically determined infiltration distance, $0.83 \mathrm{~cm}$.

Figure 9(b) shows the thermal profiles in the vicinity of the temperature peaks. The rate of the initial steep temperature rise decreases in the following order: $120 \mathrm{~K} \mathrm{~s}^{-1}$ for TC1 (located $0.3 \mathrm{~cm}$ from the silicon-carbon contact surface), 60 $\mathrm{K} \mathrm{s}^{-1}$ for TC2 $(0.5 \mathrm{~cm}), 55 \mathrm{~K} \mathrm{~s}^{-1}$ for TC3 $(0.8 \mathrm{~cm})$, and $25 \mathrm{~K} \mathrm{~s}^{-1}$ for TC4 $(1.1 \mathrm{~cm})$. It is interesting to note that the peak temperatures show a decrease with the increasing 


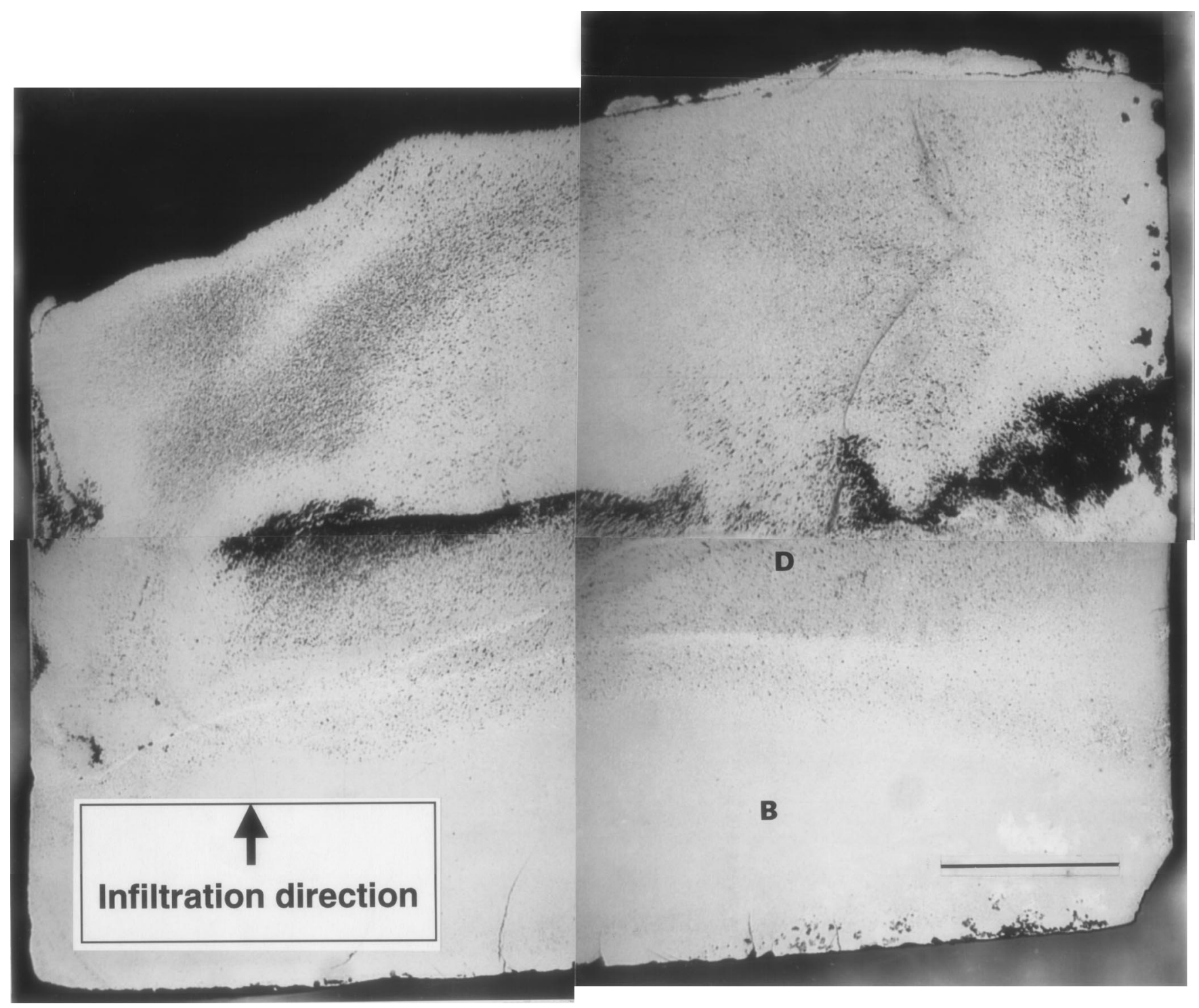

Fig. 8-Longitudinal (parallel to the infiltration direction) sections through the infiltrated B specimen (melt temperature $1743 \mathrm{~K}$ ). The dark contr ast regions are marked $\mathrm{D}$, and the light contrast regions are marked $\mathrm{B}$.

distance from the exposed surface of the preform. This is due to the continuous decrease in the infiltration front velocity as it travels into the preform, as predicted by the capillary flow analysis. Such thermal profiles can be used to estimate the infiltration front velocity for a quantitative comparison with theoretical predictions. The peak temperatures registered by the thermocouples, $2349 \mathrm{~K}$ (TC1), $2052 \mathrm{~K}$ (TC2), $1940 \mathrm{~K}$ (TC3), and $1859 \mathrm{~K}$ (TC4), also show a decreasing trend with the distance from the bottom surface.

\section{Type B preform}

The thermal profiles obtained during the infiltration of type B preforms for the same conditions used for type A preforms (i.e, melt temperature $1788 \mathrm{~K}$ ) are shown in Figures 10(a) through 10(c). The thermal responses of the four thermocouples embedded in the preform indicate that the initial temperature-rise rates, the peak temperatures, the energy evolved during the chemical reaction, and the time for the isothermal hold during the subsequent cooling are all significantly larger than those corresponding to the experiments with type A preforms (Figures 9(a) through 9(c)). For example, the rates of initial temperature rise for the TC1 through TC4 thermocouples in the B preform are 130, 175, 180 , and $50 \mathrm{~K} \mathrm{~s}^{-1}$ (Figure 10(a)), as compared with 120, 60,55 , and $25 \mathrm{~K} \mathrm{~s}^{-1}$ for type A (Figure 9(a)), respectively. The maximum temperatures registered by the four thermocouples are 2429, 2408, 2299, and $2245 \mathrm{~K}$ for type B (Figure 10 (b)), as compared with $2349,2052,1940$, and $1859 \mathrm{~K}$ for type A (Figure 9(b)). The larger extent of infiltration of the type B preform is also indicated by the considerably longer isothermal hold during cooling of the preform after the induction power is switched off; i.e., 90 (B) vs 55 (A) seconds (Figures 10(c) and 9(c)). The thermal environment for the type A and B preforms appears to be the same, as evidenced by the similar cooling rates, approximately $50 \mathrm{~K} \mathrm{~min}^{-1}$, experienced after the induction power is switched off (Figures $9(\mathrm{c})$ and $10(\mathrm{c})$ ). 


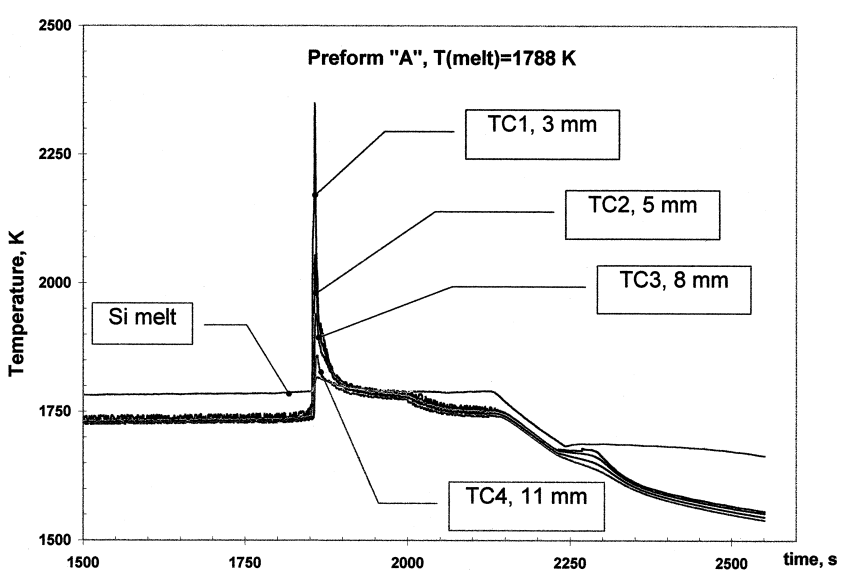

(a)

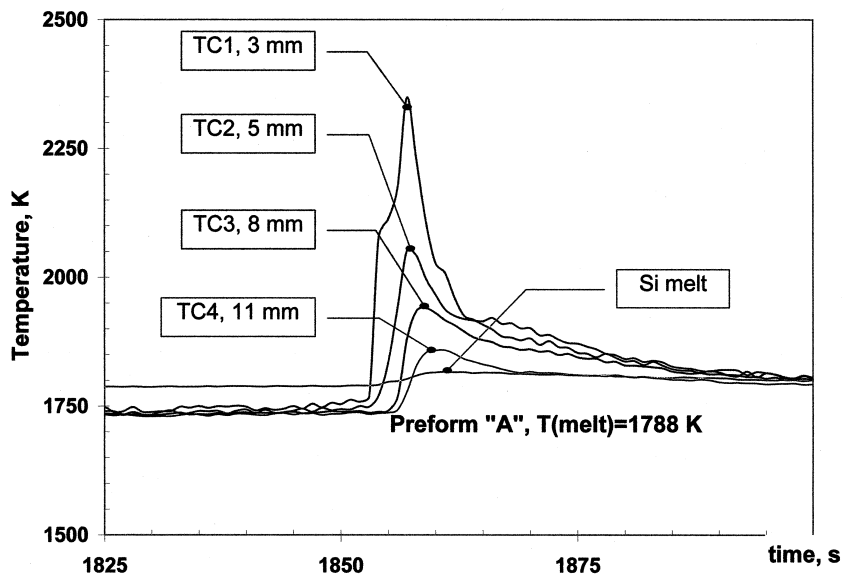

(b)

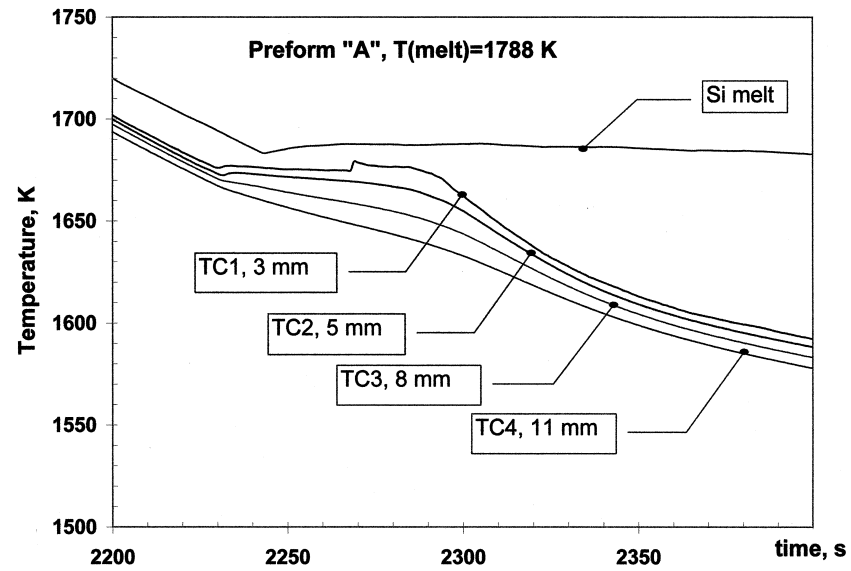

(c)

Fig. 9-Thermal profiles recorded by the thermocouples located at $0.3,0.5,0.8$, and $1.1 \mathrm{~cm}$ (TC1, TC2, TC3, and TC4, respectively) from the bottom surface of the type A preform, which was exposed to the silicon melt at $1788 \mathrm{~K}$. The silicon melt profile is indicated by "Si." (a) Overall thermal profile, (b) thermal profiles in the vicinity of exothermic peaks, and $(c)$ thermal profile during cooling.

\section{Typical thermal profiles in the presence of side} infiltration

Figure 11 shows the typical thermal profiles obtained in the presence of side infiltration due to the failure of boronnitride coating. The temperature scale here is indicated in $\mathrm{mV}$ because of the presence of thermal signals that are beyond the calibration limit of the thermocouple material. The propagating reaction pattern corresponding to unidirectional infiltration of the silicon melt (Figures 9 and 10) is no longer observed. Instead, the thermal responses resemble more a chaotic propagation pattern. Here, the melt infiltrates from the side and from the bottom, causing an almost complete infiltration of the preform and its conversion into silicon carbide. This was experimentally observed.

\section{DISCUSSION}

The analysis of capillarity-driven flow in a single pore can be used to describe flow though a porous medium, instead of using an overall characterization parameter (the medium permeability $\kappa$ ). For a single pore, the equations can be easily formulated based on Newton's law. For "highly" viscous fluids and for "short" infiltration lengths (neglecting end effects and body forces), the following closed-end form solution can be derived ${ }^{[12]}$

$$
\begin{aligned}
z^{2}= & 2\left[\frac{D_{p}^{2} \rho_{f}}{16 \mu_{f}}\right]^{2}\left[1-\exp \left(-\frac{32 \mu_{f}}{D_{p}^{2} \rho_{f}} t\right)\right] \\
& +\left[\frac{D_{p}^{2} \rho_{f}}{16 \mu_{f}}\right]\left[\frac{4 \gamma_{L V} \cos (\theta)}{D_{p} \rho_{f}}-g z\right] t
\end{aligned}
$$

which, for short times, $t \ll D_{p}^{2} \rho_{f} / 32 \mu_{f}$, becomes the following:

$$
z^{2}=\frac{D_{p}^{2} \rho_{f}}{16 \mu_{f}}\left(\frac{4 \gamma_{L V} \cos (\theta)}{D_{p} \rho_{f}}-g z\right) t
$$

here, $z$ is the coordinate along the pore axis, $\gamma_{L V}$ represents the liquid-vapor surface energy, $\theta$ is the wetting angle, and $D_{p}$ is the pore diameter, while $\rho$ and $\mu$ stand for the fluid density and dynamic viscosity, respectively.

For most cases where the flow occurs due to capillary pressure, the fluid weight is negligible at the early stages of the infiltration (i.e., for short infiltration lengths) when compared to the surface tension term, and Eq. [4] becomes the following: 


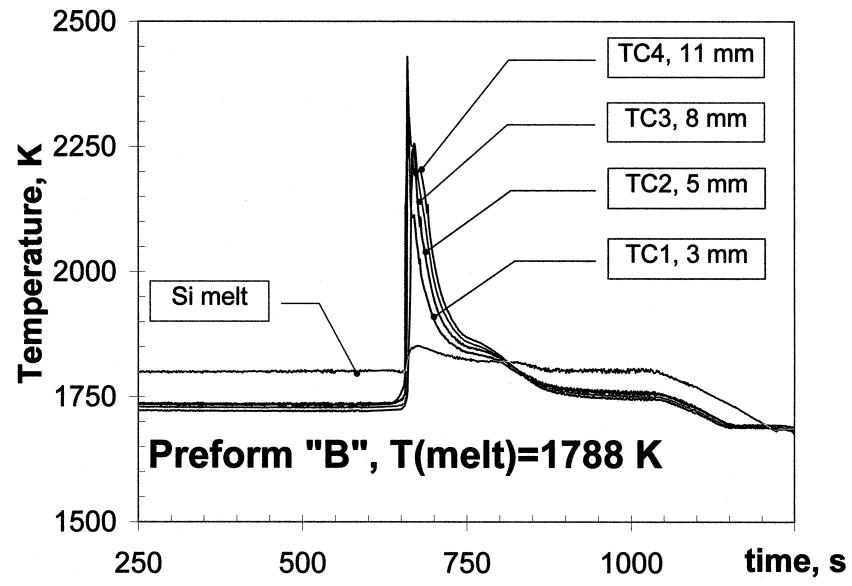

(a)

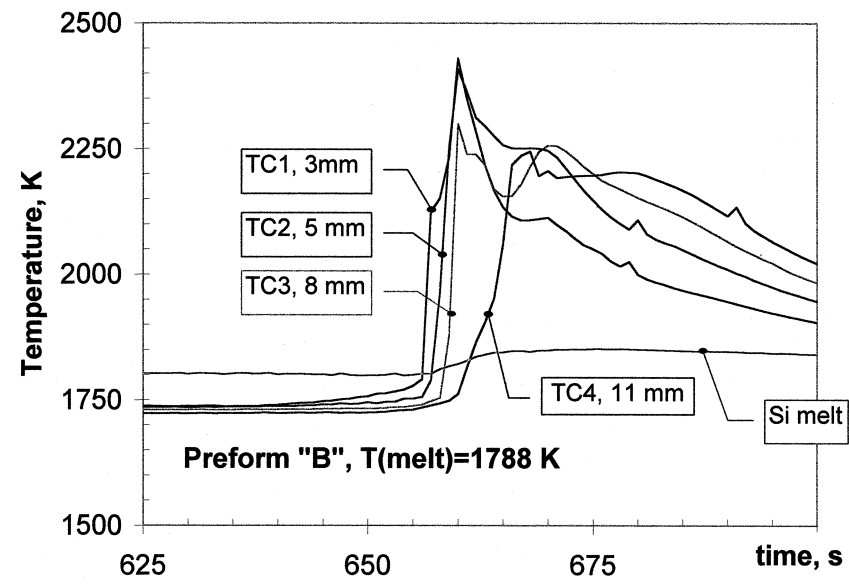

(b)

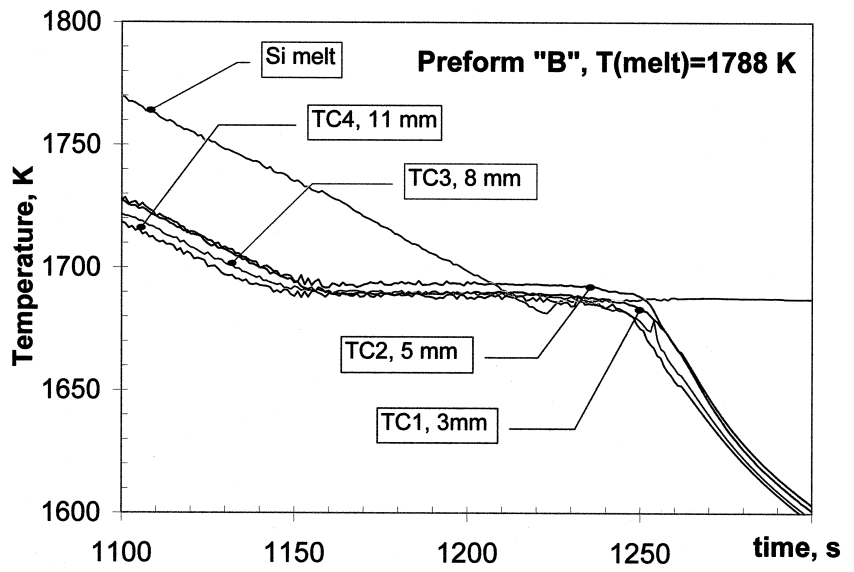

(c)

Fig. 10-Thermal profiles recorded by the thermocouples located at $0.3,0.5,0.8$, and $1.1 \mathrm{~cm}$ (TC1, TC2, TC3, and TC4, respectively) from the bottom surface of the type B preform, which was exposed to the silicon melt at $1788 \mathrm{~K}$. The silicon melt profile is indicated by Si. (a) Overall thermal profile, (b) thermal profiles in the vicinity of exothermic peaks, and $(c)$ thermal profile during cooling.

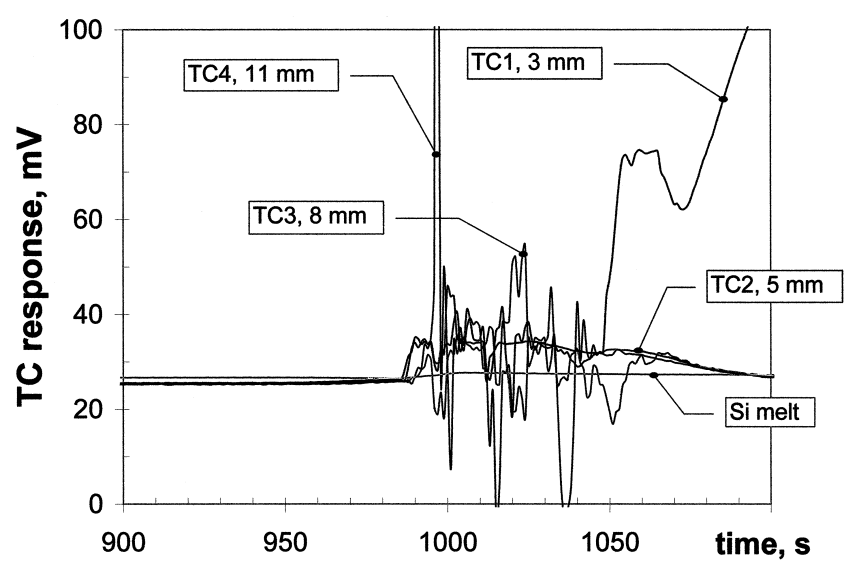

Fig. 11-Thermal responses in the presence of side infiltration (type B preform, infiltrated with Si melt at $1788 \mathrm{~K}$ ).

$$
z^{2}=\Phi t \quad \text { or } \quad v=\frac{\Phi}{2} \frac{1}{z}
$$

with

$$
\Phi=\frac{D_{p} \gamma_{L V} \cos \theta}{4 \mu_{f}}
$$

The parameter $\Phi$, often termed as "intrinsic infiltration rate" ${ }^{[13]}$ can be obtained from the experimental data by plotting infiltration length or infiltration velocity $v s$ infiltration time. One should notice, however, that Eq. [5] has been derived for flow through a single pore, and is strictly valid for a porous structure of parallel pores of diameter $D_{p}$. However, the experimental data will yield an overall $\Phi$ that is representative of the pore network (pore size, shape, distribution, and microstructure) being infiltrated. The $D_{p}$ in the equation for $\Phi$ should therefore represent an "effective pore radius".

The front velocity was estimated by following the displacement of the inflection point in the temperature profile. The time response of the different thermocouples was differentiated and estimates of the front velocity were thus obtained. Figures 12(a) and 12(b) show infiltration data and the least-squares results (fitting and 95 pct confidence intervals) corresponding to three infiltration experiments. The silicon melt was kept at a constant temperature of $1788 \mathrm{~K}$ for the three experiments reported. The experiments for type B preforms correspond to two different thermocouple arrangements: one of the experiments was performed with the four-thermocouple arrangement shown in Figure 1, while a second experiment was performed with a six-thermocouple 

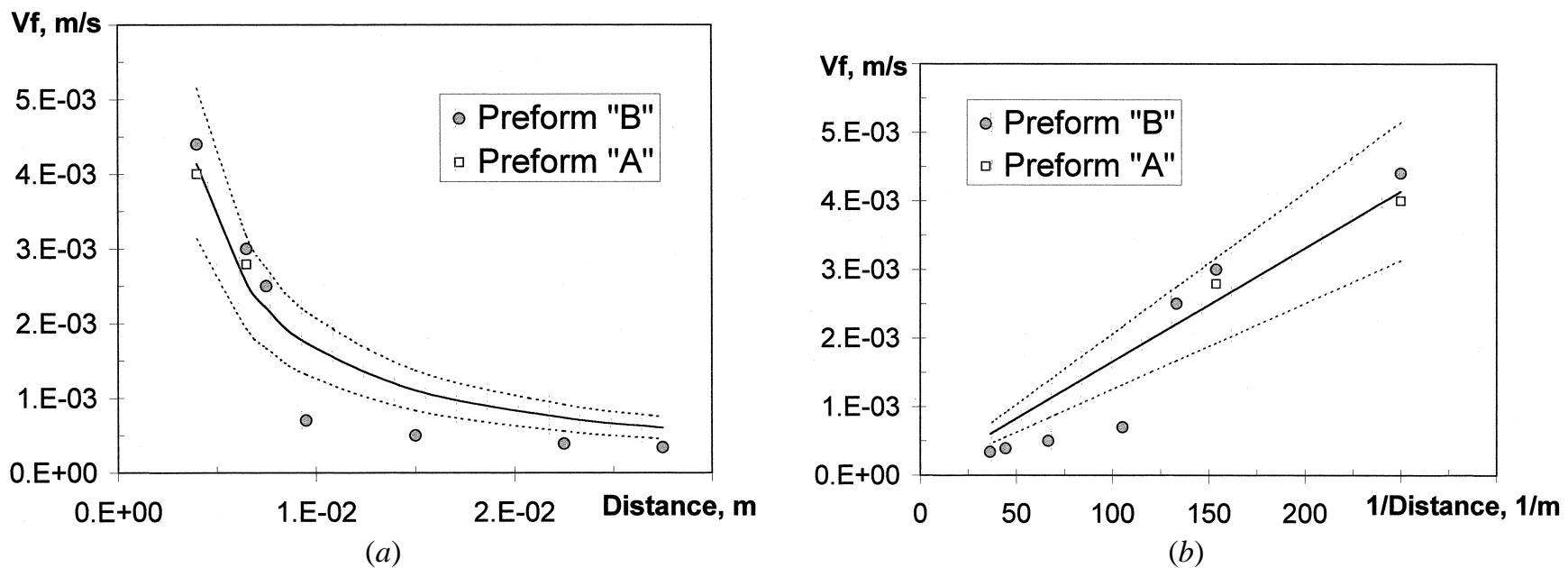

Fig. 12-Decrease in the infiltration front velocity with increasing infiltration distance: (a) nonlinear correlation and (b) linear correlation (Eq. [5]).

arrangement. The experiment for the preform type A corresponds to the four-thermocouple arrangement. As anticipated by the capillary-flow analysis, the infiltration velocity decreased with the increasing infiltration distance (Figure 12(a)). Least-squares analysis of the data in Figure 12(b) yields an approximate slope of $2 \times 10^{-5} \mathrm{~m}^{2} \mathrm{~s}^{-1}$. One could assume the pore diameter, $D_{p}$, as the volume-based median pore diameter, $1.25 \mu \mathrm{m}$ (Table I), the liquid-vapor surface energy for silicon melt, $\gamma_{L V}$, to be 0.72 to $0.76 \mathrm{~N} \mathrm{~m}^{-1}$, ${ }^{5]}$ the wetting angle (for $\mathrm{C}$ in vacuum), $\theta$, to be between 0 and $22 \mathrm{deg},{ }^{[14]}$ and the viscosity of silicon, $\mu_{f}$, to range from 0.46 to $0.76 \times 10^{-3} \mathrm{~Pa} \mathrm{~s}^{[15]}$ Then, according to Eq. [5], the intrinsic infiltration rate, $\Phi$, will be 1.37 to $2.58 \times 10^{-4} \mathrm{~m}^{2}$ $\mathrm{s}^{-1}$. This, for a preform porosity of 0.53 (Table I), will correspond to a theoretical slope ranging from 2.59 to $4.87 \times 10^{-4} \mathrm{~m}^{2} \mathrm{~s}^{-1}$. Considering that the aforementioned analysis dealt with a single pore representation of a structure consisting of a randomly oriented array of multisize pores, with the pores being assumed to remain unaltered during the infiltration process, the difference between the estimated and experimental slope values is not surprising. The occurrence of the exothermic chemical reaction has an accelerating effect on the infiltration process, which is consistent with the evidence of free silicon found for both types of preforms.

It is important to remember that any meaningful model of silicon infiltration through microporous carbon must account for the exothermic nature of the chemical reaction, and the accompanying changes in the pore morphology as a result of the chemical reaction. As was mentioned previously, the chemical reaction between the infiltrating silicon melt and carbon causes reduction in the pore size, while the temperature changes will alter physical properties and accelerate the chemical reaction. This would affect the melt flow through the pores, resulting in a different correlation between the infiltration front velocity and the infiltration distance. The two phenomena would then become intimately related as the chemical reaction, heat evolved, pore morphology, and capillary flow will interact with each other. For instance, Figure 13 shows the maximum temperature recorded by the thermocouples inserted in the preforms, plotted against the thermocouple location (infiltration distance). The peak temperatures decrease for both the preforms, with the steepest

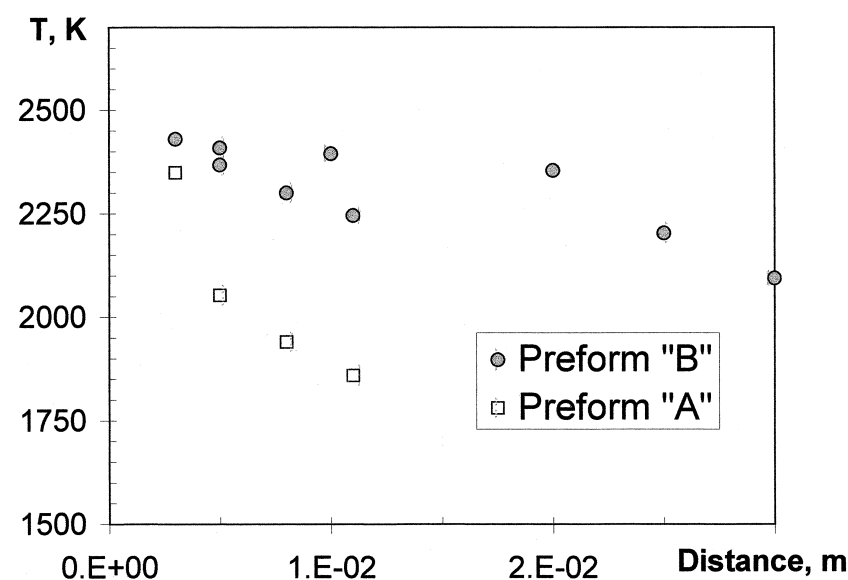

Fig. 13-Maximum temperatures as a function of infiltration distance.

decrease corresponding to the experiments in type A preforms. This indicates a higher propensity to pore closing for type A preforms. This was also observed from the metallographic examination of the infiltrated A and B specimens. However, examination of their pore characteristics (Table I) does not show any glaring differences between the two preforms. The only major differences are the higher permeability of B $\left(2.7\right.$ vs $\left.2.3 \times 10^{-14} \mathrm{~m}^{2}\right)$ and its smaller pore area per unit pore volume $\left(2.93\right.$ vs $\left.3.64 \times 10^{7} \mathrm{~m}^{-1}\right)$. One would, therefore, expect a larger extent of chemical reaction between carbon and silicon melt for the type A preform as compared with type B preforms; i.e., A would be more prone to exhibit pore closure. The capillarity-driven flow analysis, however, would suggest similar behavior for both type A and $\mathrm{B}$ preforms.

These observations indicate the need for a dynamic percolation model that would include the structural changes in the pores. The fluid contained within the microchannels (1 to $5 \mu \mathrm{m}$ ) will interact thermally with the solid skeleton, and the local fluid properties would change drastically with the sharp temperature rise. The species distribution will also be influenced. Structural changes are local phenomena, strongly 
influenced by the temperature and species distribution. Therefore, any predictive tool must include the dynamics of the flow environment, thermal interaction between the melt and solid, the temperature effects on the siliconizing process, and the "after burning" of the free silicon entrapped ahead of the choking front. In other words, a model based purely on flow analysis considerations, even when the pore structures were adapted dynamically to account for the occurrence of a chemical reaction, would not be adequate for predicting the infiltration dynamics.

\section{ACKNOWLEDGMENTS}

Appreciation is expressed to Pat Dickerson, Dave Epperly, Jim Barker, and Christopher Palda, for their technical assistance, and to Thomas K. Glasgow, Chief, Processing Science and Technology Branch, NASA-Lewis Research Center, for partial support of this research. One of the authors (JEG) gratefully acknowledges support from the Established Full-Time Faculty Research Development (EFFRD) program at Cleveland State University. The authors also thank the reviewers for their comprehensive review and suggestions.

\section{REFERENCES}

1. R.L. Mehan: J. Am. Ceram. Soc., 1977, vol. 60 (3-4), pp. 177-78.

2. Y.M. Chiang, R.P. Messner, C.D. Terwilliger, and D.R. Behrendt: Mater Sci. Eng., A144, pp. 63-74 (1991).

3. M. Singh, and D.R. Behrendt: "Studies on the Reactive Melt Infiltration of Silicon and Silicon-Molybdenum Alloys in Porous Carbon," NASA TM-105860, 1992.

4. E. Fitzer and R. Gadow: NASA, Washington, DC, Proc. Int. Symp. on Ceramics for Engines, Japan, S. Somiya, ed., 1983, pp. 561-72.

5. W.P. Minnear: Comm. Am. Cer. Soc., 1982, Jan., pp. C-10-C-11.

6. J.N. Ness and T.F. Page: J. Mater. Sci., 1986, vol. 21, pp. 1372-97.

7. T. Hase, H. Suzuki, and T. Isekei: J. Nucl. Mater., 1976, vol. 59, pp. 42-48.

8. P. Pampuch, E. Walasek, and J. Bialoskorski: Cer. Int., 1986, vol. 12, pp. $99-106$.

9. R.P. Messner and Y.M. Chiang: J. Am. Ceram. Soc., 1990, vol. 73 (5), pp. 1193-1200.

10. H.P.G. Darcy: "Les fontaines publiques de la ville de Dijon, Exposition et application des principles a suivre et des formules a employer dans les questions de distribution d'eau," Victor Dalmont, Paris, 1856.

11. E.O. Einset: J. Am. Ceram. Soc., 79(2), 333-38.

12. G.P. Martins, D.L. Olson, and G.R. Edwards: Metall. Trans. B, 19B, pp. 95-101. (1988).

13. W.B. Hillig: Cer. Bull., 73(4), pp. 56-63. (1994).

14. T.J. Whalen and A.T. Anderson: J. Am. Cer. Soc., 1975, vol. 58 (9-10), pp. 396-99.

15. B.M. Turovskii and I.I. Ivanova: Z. Akad. Nauk SSSR, Neorg. Mater, 1974 , vol. 10 (12), pp. 2108-11. 